\title{
Effectiveness of nebulised salbutamol administered in ambulances to patients with
} severe acute asthma

\author{
R J Fergusson, C M Stewart, C G Wathen, R Moffat, G K Crompton
}

\begin{abstract}
Background - Nebulised salbutamol can now be administered by ambulance personnel to patients with severe acute asthma en route to hospital. This treatment, however, is not yet available in all ambulances. The safety and effectiveness of allowing ambulance crews to initiate treatment with nebulised salbutamol has been assessed in patients with acute severe asthma.

Methods - After a basic training course in the assessment of asthma and the use of a nebuliser, ambulance crews initiated treatment with nebulised salbutamol in asthmatic patients under the age of $\mathbf{4 0}$ years. Airflow obstruction was measured before and after treatment with a peak flow meter. A subjective assessment of any change in the patient's condition was also made.

Results - Nebuliser treatment was associated with a significant increase in peak flow in almost $80 \%$ of patients who had recordable values before and after treatment. The mean percentage increase in peak flow was $56 \cdot 5 \%$. Subjective assessments correlated well with peak flow measurements. No unwanted side effects were recorded.

Conclusions - Nebulised salbutamol is an effective and safe treatment for acute asthma when administered by ambulance personnel after a short training course.

(Thorax 1995;50:81-82)
\end{abstract}

Keywords: salbutamol, emergency, asthma, ambulance.

Nebulised $\beta_{2}$ agonists are established as effective and safe treatment for patients with severe acute asthma requiring admission to hospital. ${ }^{1}$ The administration of a nebulised $\beta$ agonist in the ambulance on the way to hospital could prevent delay in treatment and may stabilise or improve a patient's condition before admission. Since 1986 all ambulances in Lothian have been equipped with nebulisers and crews have administered salbutamol in oxygen to patients with acute asthma en route to hospital. Data from the first three years of the service showed that this treatment is safe and the subjective assessment of the patient's response by ambulance personnel indicated improvement in $80 \% .^{2}$ In this study we have made an objective assessment of the effectiveness of this therapy by measuring the degree of airflow obstruction before and after nebulised treatment in the ambulance.

\section{Methods}

No age limits were set for inclusion in the study. Ambulance personnel were allowed to initiate nebuliser therapy in asthmatic patients under 40 years of age who had not been seen by a medical practitioner, and to patients who were members of the asthma self-admission services of the Edinburgh respiratory units. ${ }^{3}$ Other patients were treated at the request of the attending general practitioner. Crews were given basic training (four hour tutorial) in the recognition and initial management of severe acute asthma. They were instructed on the administration of salbutamol via a nebuliser and the measurement of airflow obstruction with a peak flow monitor. Salbutamol $(5 \mathrm{mg})$ was nebulised via a Unicorn nebuliser (System 22) using an oxygen flow rate of $61 / \mathrm{min}$. The dose of salbutamol was reduced at the discretion of the general practitioner. The peak expiratory flow (PEF) was recorded before and after completion of treatment using a Vitalograph peak flow monitor (best of three attempts). A subjective assessment of any change in the condition of the patients was also made by the ambulance crew. This involved assessing the degree of respiratory disease and the ability to converse. The condition of the patients was recorded as improved, worse, or unchanged.

\section{Results}

Data from 131 treatments in 121 patients (58 men) were recorded. The mean age was 38 years (range 2-83). Two children under the age of 12 were included. In 50 patients the treatment was initiated by the ambulance crew, in 57 by the general practitioner, and 14 were treated automatically as members of the selfadmission services. The dose of salbutamol was reduced to $2.5 \mathrm{mg}$ by the general practitioner in 15 patients including the two children.

In 20 patients it was not possible to record PEF values either before or after treatment due to respiratory distress. Seventeen could not register a reading before treatment but improved sufficiently to record their PEF afterwards. Eight patients were unable to perform PEF tests after nebulised therapy but in seven this was due to the fact that their treatment had not been completed at the time of arrival at hospital. There were 86 patients with recordable PEF values before and after nebulisation. In this group the mean pretreatment PEF was $139 \mathrm{l} / \mathrm{min}$ (95\% CI 125 to 154$)$ and after treatment it was $202 \mathrm{l} / \mathrm{min}(95 \%$ CI 180 to 223) (paired $t$ test, $t=8.41, \mathrm{p}<0.001$ ). The 
mean percentage increase in PEF was $56.5 \%$ (95\% CI $39 \cdot 5$ to $73 \cdot 5)$. Sixty eight patients (79\%) had a $>10 \%$ increase in PEF and in 14 $(16 \%)$ there was no change. In four $(5 \%)$ there was a $>10 \%$ fall in PEF but all made an uneventful recovery after admission to hospital. Subjective assessments by ambulance personnel (131 treatments) showed $79 \%$ improved, $18 \%$ unchanged, and $3 \%$ worse. No unwanted side effects from nebuliser therapy were recorded. All patients were discharged uneventfully from hospital.

\section{Discussion}

Most patients with acute severe asthma given nebulised $\beta$ agonist treatment in an ambulance en route to hospital gain a significant improvement in airflow obstruction as measured by PEF. This supports subjective data on the response of patients obtained previously from ambulance personnel. ${ }^{245}$ Our protocol allowed crews to treat patients with asthma under the age of 40 who had not been seen by a medical practitioner. The age limit was set in an attempt to avoid the administration of high flow oxygen to patients with chronic bronchitis and emphysema who may have abnormal ventilatory responses. No adverse effects were seen and it is possible that delays in delivering an effective treatment were avoided. Giving a nebulised $\beta$ agonist in an ambulance has advantages over using an air driven nebuliser in the patients' homes before the journey to hospital. Transfer to hospital is not delayed which may be vital in rural areas where long distances may need to be travelled to the nearest hospital. In addition, the drug can be nebulised in oxygen which reduces the risk of increasing hypoxaemia which may occur in patients with severe asthma when bronchodilators are nebulised in air.

Our data show that salbutamol nebulised in high flow oxygen is an effective and safe treatment for acute asthma when administered by ambulance personnel. We would encourage all ambulances to be equipped with this simple, cheap and possibly life saving facility.

1 British Thoracic Society, Research Unit of the Royal College of Physicians of London, King's Fund Centre, National Asthma Campaign. Guidelines for management of asthma in adults: II - Acute asthma. BMF 1990;301:797-800.

2 Wathen CG, Crompton GK, Carrington D, Hollingworth J. Treatment of acute asthma in the ambulance. Br $\mathcal{F} \mathrm{Gen}$ Pract 1990;40:388.

3 Crompton GK, Grant IWB. Edinburgh emergency asthma admission service. $B M \mathcal{F} 1975 ; 4: 680-2$.

4 Hill LS. The use of nebulised salbutamol by ambulance personnel. Thorax 1987;42:719.

5 Murphy S. Emergency nebulised salbutamol and the ambulance service. Scott Med f 1987;32:93.

\title{
District nebuliser compressor service: reliability and costs
}

\author{
Mary E Dodd, Simon P Hanley, Susan C Johnson, A Kevin Webb
}

\begin{abstract}
Background - There is little information on the costs of maintaining a district nebuliser compressor service. This retrospective study examines the issue, reliability, and maintenance costs of electrical compressors to assist the prediction of future costs, taking into account recent safety legislation.

Methods - Records of issue, repair, and replacement for the period 1982-91 were reviewed. The current policy of repairing and replacing as necessary, and three other theoretical costings, were considered.

Results - The number of compressors being issued is increasing. Repaired compressors are less reliable and frequency of repair is a function of compressor age. The current policy is the most cost effective.

Conclusions - To repair and replace nebuliser compressors as necessary is the most economical policy under the present terms offered by the manufacturers, but changes in safety legislation will affect the provision of such services. (Thorax 1995;50:82-84)
\end{abstract}

Keywords: outpatient, nebuliser, cost.
North Manchester Health Authority (NMHA) has experienced an increasing demand for the provision of electrical compressors used to power nebulisers for the delivery of drugs in the treatment of lung disease. It is the statutory responsibility of the Health Authority to maintain equipment provided as part of NHS treatment. ${ }^{1}$ Recommendations for servicing have been issued by the British Thoracic Society ${ }^{2}$ and general guidelines on electrical safety by the Department of Health. ${ }^{3}$ However, there are no data available on the reliability of compressors, and the merits of "regular service" compared with "as necessary maintenance" (repair including service at time of repair) on which to base recommendations.

Our policy for a 10 year period has been to repair and replace as necessary. Compressors are "replaced as necessary" if considered to be beyond economical repair by the manufacturer or agent's definition (usually parts and labour costs in excess of $£ 25$ ).

The data presented in this study are an analysis of our compressor service, examining aspects of development, repair, and replacement, in an attempt to develop a model which may be applied to a population of compressors to allow for prediction of future costs. 